\title{
SURFACTANT PROTEINS A AND B (SP-A AND SP-B) IN BRONCHOALVEOLAR LAVAGES OF CHILDREN WITH CHRONIC LUNG DISEASE
}

\author{
M. Facco ${ }^{1}$, P.E. Cogo $^{2}$, M. Simonato ${ }^{1}$, G. Lamonica ${ }^{1}$, E. Santacatterina ${ }^{1}$, E. Baraldi ${ }^{1}$, A. Baritussio ${ }^{3}$ \\ ${ }^{1}$ Department of Pediatrics, University of Padova, Padova, ${ }^{2}$ Department of Pediatric Cardiology and \\ Cardiosurgery, Bambino Gesù Children's Hospital, Roma, ${ }^{3}$ Department of Medical and Surgical Sciences, \\ University of Padova, Padova, Italy
}

Background: While surfactant protein composition of bronchoalveolar lavage (BAL) has been described in lung diseases of adults and premature infants, scanty data are available on surfactant protein composition beyond the neonatal period.

Objective: To measure SP-A and SP-B in BALs of children with chronic lung disease and to compare them with controls.

Methods: We recruited 12 children undergoing bronchoscopy for chronic lung disease (CLD group, median age 2,5 months, IQR 1,3-6,6) and 8 children who had bronchoscopy for laringotracheomalacia assessment (median age 15,7 months, IQR 11,7-19,8). BALs were collected and SP-B and SP-A amounts were quantified by ELISA using a rabbit antiserum directed against SP-B and SP-A, respectively. Data were compared by Mann-Whitney test, and a $\mathrm{p}<0.05$ regarded as statistically significant.

Results: SP-A levels were significantly lower in CLD children compared to controls (median 1,49\% of phospholipid amount (PL), IQR 0,48-6,00 versus $11,50 \%$ PL, IQR 5,04-15,05, p=0,003, in the CLD group and in controls, respectively). SP-B was measured in all 12 CLD patients and in 2 controls (analysis in progress). SP-B levels tended to be lower in the CLD group (median 0,56\% PL, IQR 0,29-4,27) compared to controls (mean 2,96\% PL, the two values being 1,80 and 4,12\% PL).

One girl in the CLD group had non detectable SP-A and SP-B levels and she was diagnosed to have ABCA3 mutation.

Conclusions: SP-A levels appear to be lower in chronic lung disease. Preliminary data suggest a similar pattern for SP-B, but this needs to be confirmed by the ongoing analysis. 